\title{
Wireless Glove for Hand Gesture Acknowledgment: Sign Language to Discourse Change Framework in Territorial Dialect
}

\author{
Shahrukh Javed ${ }^{1 *}$, Ghousia Banu $\mathrm{S}^{1}$, J Aarthy Suganthi Kani ${ }^{1}$ and Ateequeur Rahman ${ }^{2}$ \\ ${ }^{1}$ Dept of Electronics and Communication Engineering, T John Institute of Technology, India \\ ${ }^{2}$ Dept of Electronics and Communication Engineering, P A College of Engineering, India
}

Submission: June 2, 2018; Published: June 26, 2018

*Corresponding author: Shahrukh Javed, Department of Electronics and Communication Engineering, T John Institute of Technology, Gottigere, Bengaluru-560083, Email: researchscholorece@gmail.com

\begin{abstract}
Generally deaf-dumb people use sign language for communication, but they find it difficult to communicate in a society where most of the people do not understand sign language. Due to which communications between deaf-mute and a normal person have always been a challenging task. The idea proposed in this paper is a digital wireless glove which can convert sign language to text and speech output. The glove is embedded with flex sensors and a 9DOF inertial measurement unit (IMU) to recognize the gesture. A 9-degree of freedom inertial measurement unit (IMU) and flex sensors are used to track the orientation of fingers and motion of hand in three dimensional spaces which senses the gestures of a person in the form of bend of fingers and tilt of the hand fist. This system was tried for its practicality in changing over gesture based communication and gives the continuous discourse yield in local dialect and additionally shows the text on GLCD module. The text show being in English, the voice yield of the glove will be in provincial dialect (here Kannada). So this glove goes about as a communicator which in part encourages them to get their necessities and an interpreter giving greater adaptability in correspondence. In spite of the fact that the glove is planned for gesture based communication to discourse transformation, it is a multipurpose glove and discovers its applications in gaming, mechanical autonomy and therapeutic field.In this paper, we propose an approach to avoid the gap between customer and software robotics development. We define a EUD (End-User Development) environment based on the visual programming environment Scratch, which has already proven in children learning computer science. We explain the interests of the environment and show two examples based on the Lego Mindstorms and on the Robosoft Kompai robot.
\end{abstract}

Keywords: Sign Language; Flex Sensors; State Estimation Method; 3D Space; Gesture Recognition

Abbreviations: ANN: Artificial Neural Networks; SAD: Sum of Absolute Difference; IMU: Inertial Measurement Unit; ADC: Analog to Digital Converter; HCI: Human Computer Interface

\section{Introduction}

About nine thousand million people in the world are deaf and mute. How commonly we come across these people communicating with the normal world? The communication between a deaf and general public is to be a thoughtful issue compared to communication between visually impaired and general public. This creates a very small space for them as communication being a fundamental aspect of our life. The blind people can talk freely by means of normal language whereas the deaf-mute people have their own manualvisual language popularly known as sign language [1]. The development of the most popular devices for hand movement acquisition, glove-based systems started about 30 years ago and continues to engage a growing number of researchers. Sign language is the non-verbal form of intercommunication used by deaf and mute people that uses gestures instead of sound to convey or to express fluidly a speaker's thoughts. A gesture in a sign language is a particular movement of the hands with a specific shape made out of them [2]. The conventional idea for gesture recognition is to use a camera based system to track the hand gestures. The camera based system is comparatively less user friendly as it would be difficult to carry around.

The main aim of this paper is to discuss the novel concept of glove based system that efficiently translates Sign Language gestures to auditory voice as well as text and also promises to be portable [3]. Several languages are being spoken all around the world and even the sign language varies from region to region, so this system aims to give the voice output in regional languages (here Kannada). For Sign language recognition few attempts have been made in the past to recognize the gestures 
using camera, Leaf switches and copper plates but there were certain limitations of time and recognition rate which restricted the glove to be portable. Mainly there were two well-known approaches viz. Image processing technique and another is processor and sensor based data glove [4]. These approaches are also known as vision based and sensor based techniques. Our system is also one such sensor based effort to overcome this communication barrier, which senses the hand movement through flex sensors and inertial measurement unit and then transmits the data wirelessly to the raspberry pi which is the main processor, that accepts digital data as input and processes it according to instructions stored in its memory, and outputs the results as text on GLCD display and a voice output.

\section{Background Work}

People who are hard of hearing or quiet are isolated in the cutting edge work environment as well as in regular daily existence making them live in their own different networks. For example, there have been enhancements in amplifiers and cochlear inserts for the hard of hearing and counterfeit voice boxes for the quiet with vocal rope harm. Be that as it may, these

Table 1: Comparison of Background Work.

\begin{tabular}{|c|c|}
\hline Different Method of Recognizing Gestures & Limitations \\
\hline CMOS camera based glove & Highly expensive and high latency \\
\hline Leaf switch based glove & $\begin{array}{l}\text { With increase in usage, the switch instead of being open when the finger is straight, it will be } \\
\text { closed resulting in improper transmission of gesture. }\end{array}$ \\
\hline Copper plate based glove & $\begin{array}{c}\text { The use of copper plate makes the glove bulky which makes it uncomfortable to use it for a } \\
\text { longer time. }\end{array}$ \\
\hline In MEMS Accelerometer based system & Portability of such systems is very difficult. \\
\hline ARM based system & It requires more number of sensors and a computer to maintain a database \\
\hline
\end{tabular}

\section{Problem Definition}

With a population of around 7.6 billion today communication is a strong means for understanding each other. Around nine thousand million individuals are deaf and mute. Individuals with discourse hindrance their vocal articulation are not reasonable, they require a specific skill like static state of the hand orientation to give a sign, more as manual-visual dialect prevalently known as sign language to communicate with general population. They think that it's hard to impart in a general public where a large portion of the general population don't comprehend sign language. Hence forth they find a little space to convey and do not have the capacity to impart at a arrangements don't come without drawbacks and expenses [5]. Cochlear inserts have even caused a tremendous debate in the hard of hearing network and numerous decline to considerably think about such arrangements. Thusly, we trust society still requires a compelling answer for expel the correspondence obstruction between hard of hearing and quiet people and nonmarking individuals. Our proposed arrangement and objective is to plan a Human Computer Interface (HCI) gadget that can make an interpretation of gesture based communication to content and discourse in provincial dialect, furnishing any hard of hearing and quiet people with the capacity to easily speak with anybody [6]. The thought is to plan a gadget put on a hand with sensors fit for assessing hand signals and after that transmitting the data to a preparing unit which plays out the communication via gestures interpretation. The last item will have the capacity to proficiently perform gesture based communication and give the focused on yield. We want to have the capacity to enhance the personal satisfaction of hard of hearing and quiet people with this gadget.

\section{Comparison of Background Related Work}

(Table 1)

Table 2: reference table.

\begin{tabular}{|c|c|c|c|}
\hline Year & Author & Title & Methodology \\
\hline 2017 & $\begin{array}{l}\text { L.Anusha, } \\
\text { Y.Usha Devi }\end{array}$ & $\begin{array}{l}\text { Implementation Of Gesture } \\
\text { Based Voice And Language } \\
\text { Translator For Dumb People }\end{array}$ & $\begin{array}{l}\text { System consists of MPU6050, Raspberry pi, three } \\
\text { button Keypad and speaker. It utilizes trajectory } \\
\text { recognition algorithm for recognizing alphabets. } \\
\text { Raspberry pi generates voice output for the text in } \\
\text { multiple languages using voice RSS and Microsoft } \\
\text { translator. }\end{array}$ \\
\hline
\end{tabular}


Robotics \& Automation Engineering Journal

\begin{tabular}{|c|c|c|c|}
\hline 2016 & $\begin{array}{c}\text { A.H.Ansari Ms.joriAshwini } \\
\text { Ms.Gaikwad }\end{array}$ & $\begin{array}{c}\text { Giving Voice To Mute People } \\
\text { Using Flex Sensor }\end{array}$ & $\begin{array}{c}\text { System utilizes Statistical Template Matching } \\
\text { Technique for sign language recognition which } \\
\text { provides accuracy comparable to those of } \\
\text { commercially available systems and that too at a } \\
\text { highly optimized cost. }\end{array}$ \\
\hline 2016 & $\begin{array}{c}\text { Kalpattu S, Abhishek, } \\
\text { Derek Ho }\end{array}$ & $\begin{array}{c}\text { Glove Base And Gesture } \\
\text { Recognition Sign Language } \\
\text { Translator Using Capacitive } \\
\text { Touch Sensor }\end{array}$ & $\begin{array}{c}\text { Charge transfer touch sensor based system } \\
\text { for translation of ASL. Capacitive touch sensor and } \\
\text { Raspberry Pi embedded system to recognize and } \\
\text { translate hand gesture in to sound. }\end{array}$ \\
\hline
\end{tabular}

\section{Proposed System}

(Figure 1)

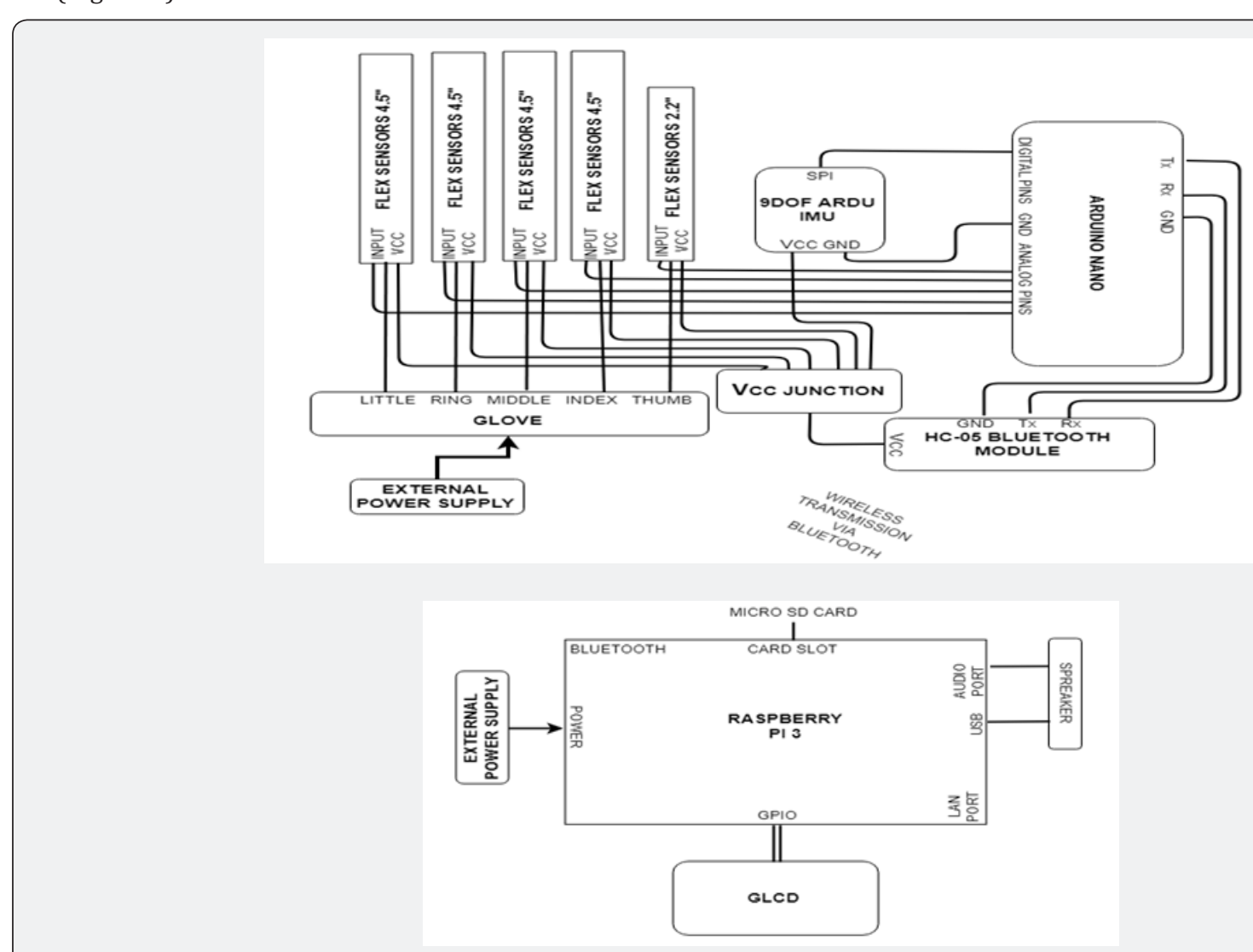

Figure 1: System Block Diagram.

\section{Block Diagram Explanation}

The sensor based system is designed using four 4.5 inch and two 2.2-inch flex sensors which are used to measure the degree to which the fingers are bent. These are sensed in terms of resistance values which is maximum for minimum bend radius. The flex sensor incorporates a potential divider network which is employed to line the output voltage across 2 resistors connected as shown in Figure 2.

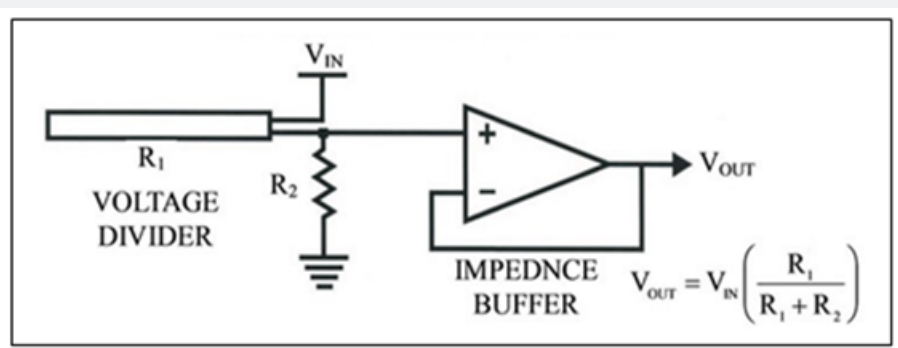

Figure 2: Equivalent circuit of flex sensor. 
The output voltage is determined using the following equation,

$$
V_{\text {out }}=\frac{V_{\text {in }} X R 1}{(R 1+R 2)}
$$

Where;

\section{R1 - flex sensor resistance. R2 - Input resistance.}

The external resistor and flex forms a potential divider that divides the input voltage by a quantitative relation determined by the variable and attached resistors. For particular gestures the current will change, as a result of the changing resistance of the flex sensor which is accommodated as analog data. One terminal of flex sensor is connected to the 3.3Volts and another terminal to the ground to close the circuit. A 9-Degree of Freedom Ardu Inertial Measurement Unit (IMU) is essential for accelerometer and gyroscope readings which is placed on the top of the hand to determine hand position. The co-related 3D coordinates are collected by the inertial measurement unit as input data [8]. The impedance values from flex sensors and IMU coordinates for individual gesture are recorded to enumerate the database. The database contains values assigned for different finger movements. When the data is fed from both flex sensors and IMU to Arduino nano it will be compute and compare with the predefined dataset to detect the precise gesture and transmitted wirelessly to the central processor i.e. raspberry pi via Bluetooth module. Raspberry Pi 3 is programed to display text output on GLCD. Graphic LCD is interfaced with the Raspberry pi3 using 20-bit universal serial bus in order to avoid bread board connection between processor and the display, and a $10 \mathrm{Kohm}$ trim potentiometer is used to control the brightness of display unit. Further to provide an auditory speech, pre-embedded regional language voice is assigned for each conditions as similar to the text database which is mapped with the impedance values. Two speakers are used with single jack of $3.5 \mathrm{~mm}$ for connection and a USB to power-up the speakers [9]. When text is displayed, the processor will search for the voice signal which will be transmitted through speakers.

\section{Methodology (Figure 3)}

a) The gesture is served as an input to the system which is measured by both the sensors particularly from the flex sensor in terms of impedance and the IMU gives the digital values.

b) These values from the flex sensor are analog in nature and is given to the Arduino nano which uses the analog to digital convertor consolidated in it to convert the resistive values to digital values.

c) IMU utilizes the accelerometer/gyroscope sensors to measure the displacement and position of the hand.

d) These qualities from both the sensors are fed to
Arduino nano which contrasts it and the values stored in the predefined database, and further transmits this digital data wirelessly to the main processor by means of Bluetooth.

e) Central processor the raspberry pi3 is coded in python dialect for processing the received digital signals to generate the text output, for example, characters, numbers and pictures. Further, the text output is shown on Graphic-LCD display and next text to speech engine, here particularly espeak converter is utilized to give the soundrelated voice output [10].

Finally, system effectively delivers the output as text and auditory voice in regional dialect.

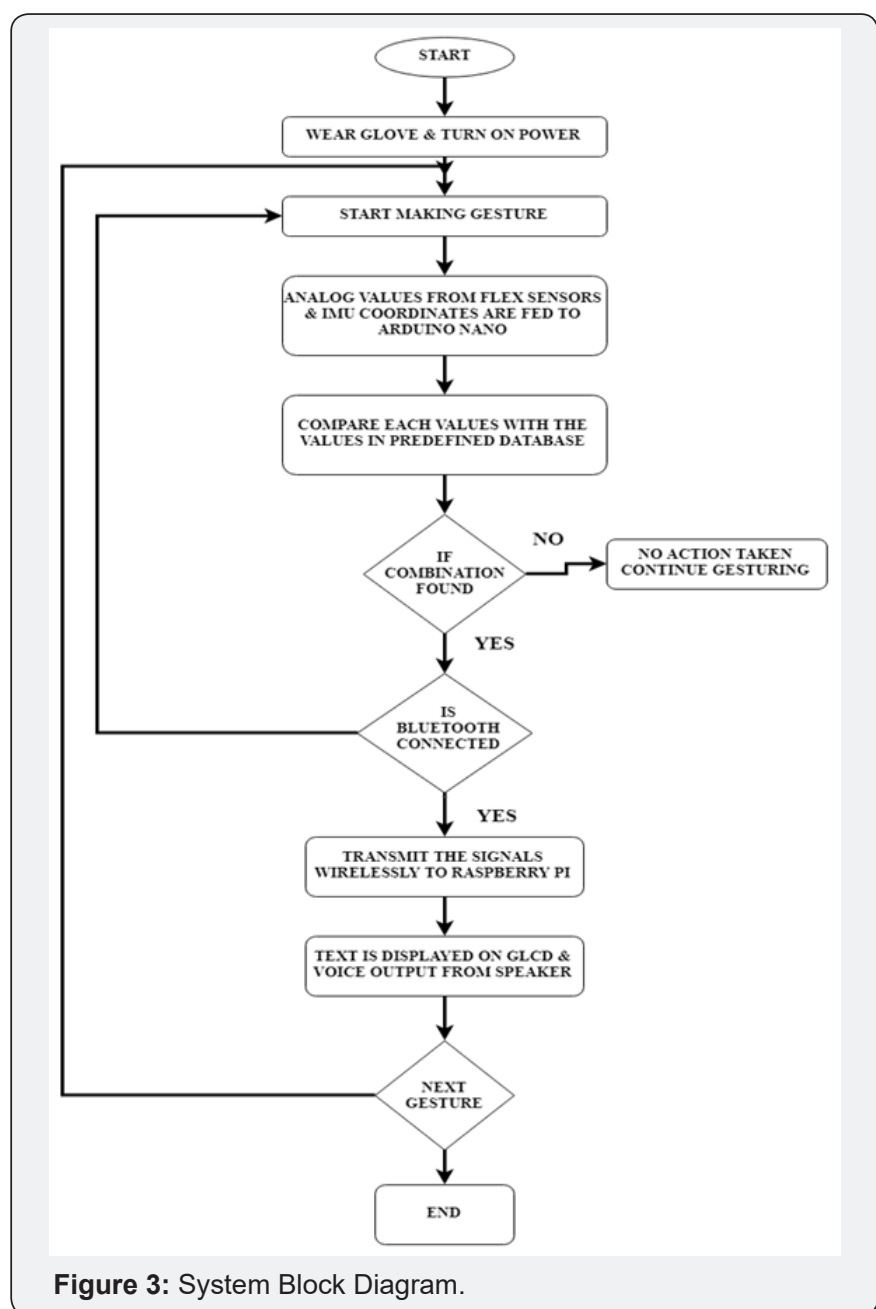

\section{Prototype Implementation and its Working}

In this system, the features are extracted from the hardware sensor inputs and the targets are the words in sign language. To realize the design requirements, several hardware components were considered during prototyping. Much of the hardware was selected due to their ease of use and accuracy. The Ardu 9DOF IMU was selected for convenience as it produces the computation necessary for linear acceleration and gyroscopic 


\section{Robotics \& Automation Engineering Journal}

measurements. The roll, pitch and yaw measurements were found to be roughly $\pm 2^{\circ}$ accuracy which is far beyond sufficient for our sign language requirements. Since the flex sensor required additional analog pins, careful planning allowed us to fit the circuit in an agreeable manner on the hand glove. Space was managed to add in the HC05 Bluetooth module onto the device. All the sensors must be placed in a way as to not make contact with each other causing short circuits which and disruption of measurement readings [11]. Electrical tape was necessary to provide insulation for our sensors. The system recognizes gestures made by the hand movements by wearing the glove on which two sensors are attached, the first sensor is to sense the bending of five fingers, the flex sensor of 2.2 inches for thumb and for the other four fingers of 4.5 inches and the second sensor used is 9-DOF Inertial Measurement Unit to track the motion of hand in the three-dimensional space, which allows us to track its movement in any random direction by using the angular coordinates of IMU (pitch, roll and yaw). Since the output of flex sensor is resistive in nature the values are converted to voltage using a voltage divider circuit.

The resistance values of 4.5-inch flex sensors range from $7 \mathrm{~K}$ to $15 \mathrm{~K}$ and for 2.2 -inch flex sensor, it ranges from $20 \mathrm{~K}$ to $40 \mathrm{~K}$, as shorter the radius the more resistor value. Another $2.5 \mathrm{~K} \mathrm{ohm} \mathrm{resistor} \mathrm{is} \mathrm{utilized} \mathrm{to} \mathrm{build} \mathrm{a} \mathrm{voltage} \mathrm{divider} \mathrm{circuit}$ with Vcc supply being 3.3volts taken from Arduino nano processor, the voltage values from the voltage divider circuit being analog in nature are given to the Arduino nano processor which has an inbuilt ADC [12]. Further, the IMU senses the hand movements and gives the digital values in XYZ direction called the roll, yaw, pitch respectively. The values from the IMU and values of the flex sensors are processed in the Arduino nano which is interfaced with HC-05 Bluetooth module embedded on the glove which provides the approximate range of 10 meters. The data processed in nano are sent wirelessly through Bluetooth to the central processor i.e. Raspberry Pi which is coded in python, in a way to convert given values into the text signal by searching in database for that particular gesture. In accordance with the digital value received, the impedance values along with 3 dimensional IMU coordinates for each individual gestures are recorded to enumerate the database. The database contains collective resistance values assigned for different finger movements. When the computed data is received by the processor, it is compared with the measured dataset to detect the precise gestures. If the values matches, then the processor sends the designated SPI commands to display the texts according to gestures onto the GLCD and the espeak provides the text to speech facility giving audible voice output in regional language through the speakers. Further, for any next gestures made, both flex sensor and IMU detects and data is compared with the database already present in the processor and if it matches, displays in text format as well as audible output speech will be given by the speakers [13] (Figure 4).

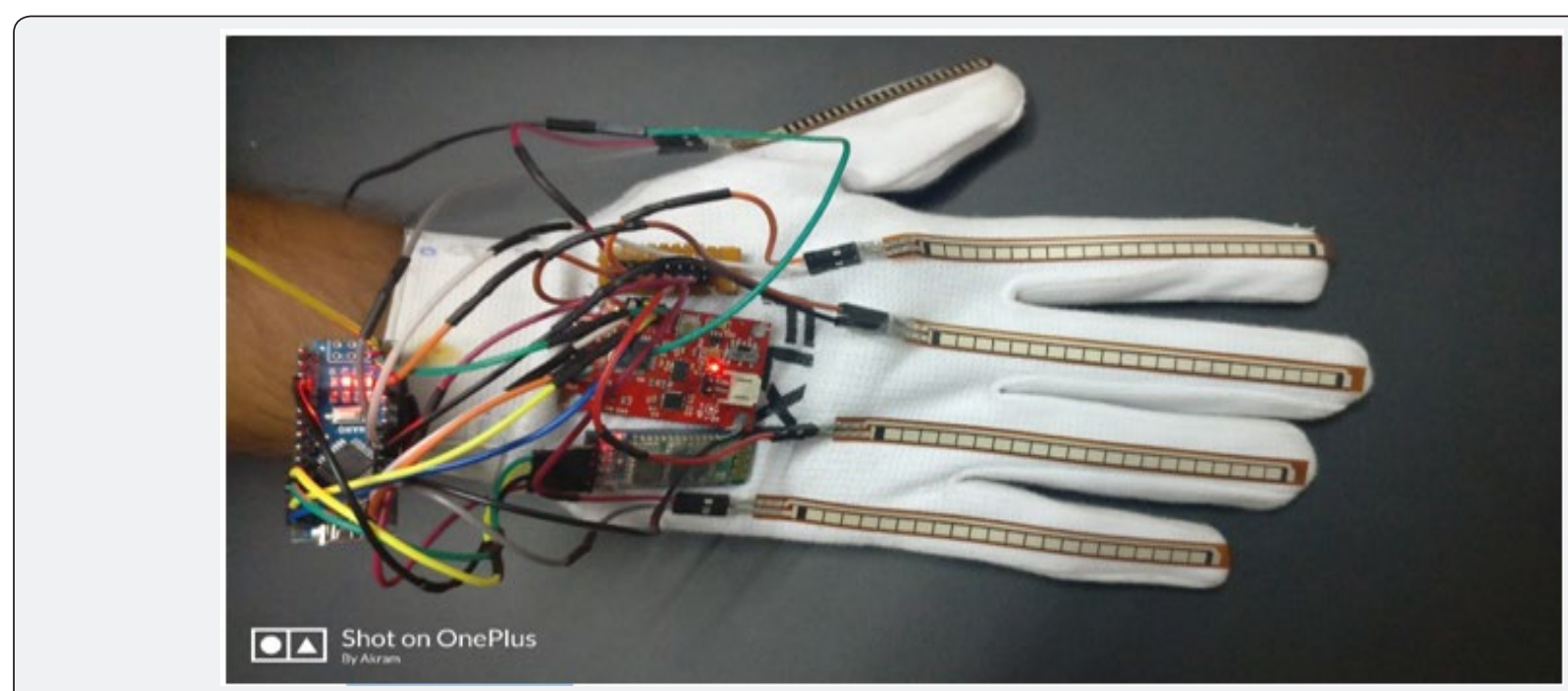

Figure 4: Prototype Module.

\section{Results}

In this prototype system, the user forms a gesture and holds it approximately for 1 or 1.5 seconds to ensure proper recognition. Each gesture comprises of bending of all fingers in certain angles accordingly. Every bend of the sensor (finger) produces unique ADC value so that when different hand gesture is made, different ADC values are produced. Taking such ADC values for 4 different users, a table of average ADC values for each sensor is maintained where F1, F2, F3, F4 and F5 represents the little finger, the ring finger, the middle finger, the index finger and thumb respectively. Table 3 shows the gestures and corresponding words voiced out. The hand signs taken in the prototype can be easily modified using the concept of ADC count according to the user convenience. At the same time the voice output can be changed easily to gives a flexibility in change of language according to different regions (Figure 5). 
Robotics \& Automation Engineering Journal

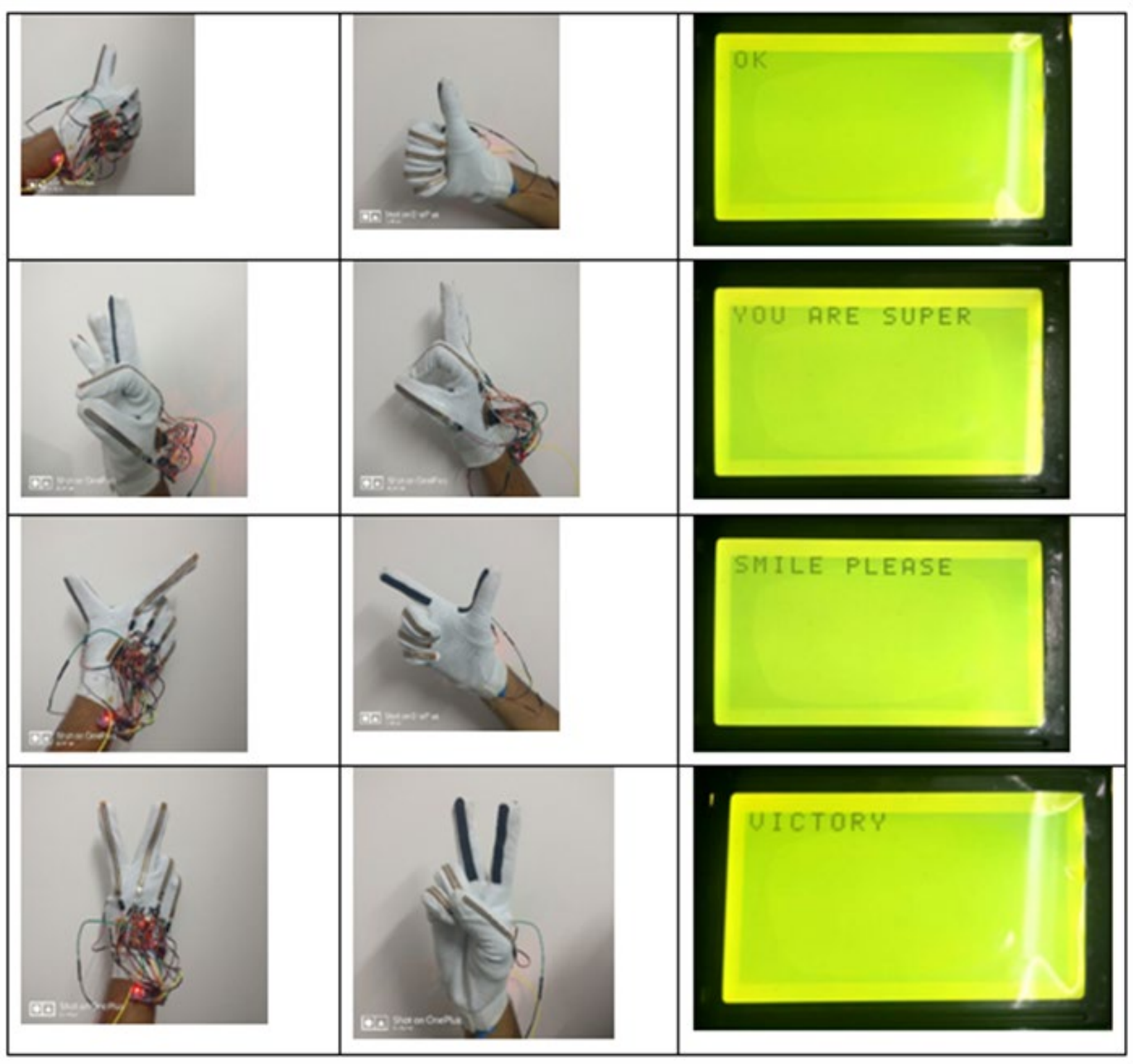

Figure 5: Some of the Hand Gestures used in the system.

Table 3: ADC values of each sensor (finger) for different gestures.

\begin{tabular}{|c|c|c|c|c|c|}
\hline Gesture & Little & Ring & Middle & Index & Thumb \\
\hline OK & $>745$ & $>682$ & $>722$ & $>725$ & $>335$ \\
\hline $\begin{array}{c}\text { YOU ARE } \\
\text { SUPER }\end{array}$ & $>740$ & $>680$ & $>700$ & $<721$ & $<335$ \\
\hline VICTORY & $<745$ & $<682$ & $>700$ & $>721$ & $<335$ \\
\hline $\begin{array}{c}\text { SMILE } \\
\text { PLEASE }\end{array}$ & $<745$ & $<682$ & $<722$ & $>720$ & $>335$ \\
\hline
\end{tabular}

Applications

a) Voice interpreter for mute people

b) No touch user interface

c) Gaming industry: Hand gestures play a vital role in the gaming industry, especially in first person shooting games. The player can control the character in the game using his hand and this could give a real life experience of the game. Also, virtual reality is gaining grounds in the gaming industry. Combining virtual reality with the gloves with a haptic feedback can give the gamer a real life gaming experience.

d) Controlling a robotic arm using the gloves: The gloves could be used to control a robotic arm. The applications for this system are wide. With incorporation of haptic feedback, the glove -robotic arm interface could also be used in bomb diffusion.

e) Remote medical surgery: In this the surgeon need not be at the physical location to perform the surgery. He could control a robotic arm remotely to perform the surgery 
and a haptic feedback could give him the feel of actually performing the surgery. But this would require the gesture recognition to be very precise and the transfer of data from the hand to the robotic arm should be without even a tiny glitch.

\section{Advantages}

a) Cost effective light weight and portable

b) Real time translation approximately in no time delay

c) Flexible for ' $N$ ' users with easy operation

d) Fully automate system for mute communication

\section{Future scope}

a) The system can be further developed with Wi-Fi connection and enlarged database supporting special characters or symbols.

b) Microsoft Text To Speech (TTS) engine can be utilized to provide compatibility for multiple international languages.

c) An Android application can be developed for displaying the text and speech output on an Android device.

\section{Conclusion}

As we discovered that Deaf-quiet individuals utilize communication via gestures to cooperate with others however numerous don't comprehend this motion dialect. We have built up a sensor based motion acknowledgment framework to undercover signal into local dialect discourse and content yield. In this framework the hard of hearing quiet individuals wear the gloves to perform hand motion, the transformation of content to discourse in provincial dialect and show has been seen to be predictable and dependable. In this way, the proposed framework with the database of 20 words and 15 sentences has been effectively created which changes over the motions into English words or sentences and shows the yield on GLCD and relating voice yield in provincial dialects using espeak. The proposed framework has insignificant equipment mounted on it which makes it dependable, convenient and savvy and more straightforward to speak with the general public [14]. One more requesting where this framework could be utilized as a part without bounds: Helping hand for individuals with Cerebral Palsy; Cerebral palsy usually appears in early childhood and involves a group of permanent movement disorders. The symptom varies with people and often includes poor coordination, stiff muscles, weak muscles, and tremors. Also, the problems with sensation, vision, and hearing, swallowing and speaking have been identified as other symptoms $[15,16]$. This problem can be solved to a great extent by providing them with a provision to communicate with just a single finger. The frequently used words by such people can be put across to people with just a small movement in the finger and using our state estimation technique it could predict the letters or words [17].

\section{References}

1. Anusha L, Usha Devi Y (2016) Implementation Of Gesture Based Voice And Language Translator For Dumb People. International Conference on Communication and Electronic Systems (ICCES) ISBN:978-1-50901066-0.

2. Abhijith Bhaskaran K, Anoop G Nair (2016) Smart Gloves for Hand Gesture Recognition Sign Language to Speech Conversion System. Department of ECE, 2016 International Conference on RAHA ISBN: 978-1-5090-5203-5.

3. Ansari AH, Ashwini Sanjay (2016) Giving Voice To Mute People Using Flex Sensor. 2016 IJARIIE-ISSN(0)-2395-4396 2(3).

4. Kalpattu S, Abhishek, Derek Ho (2016) Glove Base And Gesture Recognition Sign Language Translator Using Capacitive Touch Sensor. IEEE International Conference on Electron Devices and Solid-State Circuits. ISBN: 978-1-5090-1830-7.

5. Jadhav BD, Nipun Munot, Madhura Hambarde, Jueli Ashtikar (2015) IJCSN International Journal of Computer Science and Network ISSN (Online) : 2277-5420

6. Aarthi M, Vijayalakshmi P (2016) Sign Language to Speech Conversion. Department of ECE Department of ECE, SSN College of Engineering SSN College of Engineering.

7. Celestine Preetham, Girish Ramakrishnan, Sujan Kumar, Anish Tamse, Nagendra Krishnapura, (2013) Hand Talk- Implementation of a Gesture Recognizing Glove. Department of Electrical Engineering, Indian Institute of Technology Madras, Bangalore, India.

8. Pooja Gupta, Dr. Ambuj Kumar Agrawal, Shahnaz Fatima (2014) Sign Language Problem And Solutions For Deaf And Dumb People. pp. 124127.

9. Sawant Pramada, Deshpande Saylee, Nale Pranita, Nerkar Samiksha, Archana S Vaidya (2013) Intelligent Sign Language Recognition Using Image Processing 3(2): 45-50.

10. Kunal A Wankhade, Gauri N Zade (2014) Sign Language Recognition For Deaf And Dumb People Using ANFIS 3(5): 1206-1209.

11. Kiratey Patil, Gayatri Pendharkar, Gaikwad GN (2014) American Sign Language Detection 4(11): 1-5.

12. Shanmukha Swamy MN, Chethan MP, Mahantesh Gatwadi (2014) Indian Sign Language Interpreter with Android Implementation 97(13): 36-40.

13. Etsuko Ueda, Yoshio Matsumoto, Masakazu Imai, Tsulasa Ogasawara (2001) Hand Pose Estimation for Vision based Human Interface. In Proc $10^{\text {th }}$ IEEE International Workshop on Robot and Human Communication (Roman 2001), pp. 473- 478.

14. Claudia Nölker, Helge Ritter (1999) Visual Recognition of Hand Postures. In Proc International Gesture Workshop on Gesture-Based Communication in Human Computer Interaction, pp. 61-72. 
15. Wilson AD, Bobick AF (2011) Learning visual behavior for gesture analysis. In Proc IEEE Symposium on Computer Vision.

16. Zhang Qiu-yu, Lu Jun-chi, Zhang Mo-Yi, Duan Hong - Xiang And Lv Lu (2015) Hand Gesture Segmentation Method Based on YCb $\mathrm{Cr}$ Color Space and K- Means Clustering. International Journal Of signal
Processing , Image Processing and Pattern Recognition 8(5): 105- 116.

17. Jorge Alberto Marcial Basilio, Gulaberto Aguilar Torres, Gabriel Sanchez Perez, Karina Toscano Medina L, Hector M Perez Meana (2011) Explicit Image Detection Using YCbCr Space Color Model as Skin Detection. Application of Mathematics and Computer Engineering, pp: 123-125.

\section{Your next submission with Juniper Publishers will reach you the below assets}

- Quality Editorial service

- Swift Peer Review

- Reprints availability

- E-prints Service

- Manuscript Podcast for convenient understanding

- Global attainment for your research

- Manuscript accessibility in different formats

( Pdf, E-pub, Full Text, Audio)

- Unceasing customer service

Track the below URL for one-step submission https://juniperpublishers.com/online-submission.php 